\title{
Breakfast glycaemic index and cognitive function in adolescent school children
}

\author{
Simon B. Cooper*, Stephan Bandelow, Maria L. Nute, John G. Morris and Mary E. Nevill \\ Institute of Youth Sport, School of Sport, Exercise and Health Sciences, Sir John Beckwith Centre for Sport, Loughborough \\ University, Leicestershire LE11 3TU, UK
}

(Submitted 12 April 2011 - Final revision received 8 August 2011 - Accepted 8 August 2011 - First published online 29 September 2011)

\section{Abstract}

It has been suggested that a low-glycaemic index (GI) breakfast may be beneficial for some elements of cognitive function (e.g. memory and attention), but the effects are not clear, especially in adolescents. Thus, the aim of the present study was to examine the effects of a low-GI breakfast, a high-GI breakfast and breakfast omission on cognitive function in adolescents. A total of fifty-two adolescents aged 12-14 years were recruited to participate in the study. Participants consumed a low-GI breakfast, a high-GI breakfast or omitted breakfast. A battery of cognitive function tests was completed 30 and 120 min following breakfast consumption and capillary blood samples were taken during the $120 \mathrm{~min}$ postprandial period. The findings show that there was a greater improvement in response times following a low-GI breakfast, compared with breakfast omission on the Stroop $(P=0 \cdot 009)$ and Flanker $(P=0 \cdot 041)$ tasks, and compared with a high-GI breakfast on the Sternberg paradigm $(P=0.013)$. Furthermore, accuracy on all three tests was better maintained on the low-GI trial compared with the high-GI (Stroop: $P=0 \cdot 039$; Sternberg: $P=0 \cdot 018$; Flanker: $P=0 \cdot 014$ ) and breakfast omission (Stroop: $P<0 \cdot 001$; Sternberg: $P=0.050$; Flanker: $P=0.014)$ trials. Following the low-GI breakfast, participants displayed a lower glycaemic response $(P<0 \cdot 001)$ than following the high-GI breakfast, but there was no difference in the insulinaemic response $(P=0 \cdot 063)$ between the high- and low-GI breakfasts. Therefore, we conclude that a low-GI breakfast is most beneficial for adolescents' cognitive function, compared with a high-GI breakfast or breakfast omission.

Key words: Breakfast: Glycaemic index: Cognitive function: Adolescents

Breakfast consumption, as opposed to breakfast omission, has a positive impact on cognitive function in adults ${ }^{(1,2)}$, children $^{(3)}$ and adolescents ${ }^{(4,5)}$. In adults, the effects of breakfasts differing in macronutrient content ${ }^{(6)}$, glycaemic load ${ }^{(7)}$ and glycaemic index $(\mathrm{GI})^{(8-10)}$ on cognition have also been examined, with evidence suggesting that low-GI foods are beneficial for some aspects of adults' cognitive function, including working memory ${ }^{(8,10)}$ and attention ${ }^{(10)}$.

Fewer studies have examined the effect of the GI of breakfast on cognitive function of young people and adolescents, and the findings have been equivocal ${ }^{(3)}$. It has been suggested that adolescent populations are particularly important to study in this field as while going through puberty, adolescents undergo rapid growth and changes in metabolism and thus their responses may be different from those of younger children and adults ${ }^{(11,12)}$. Furthermore, the academic work completed by adolescents is of a greater complexity than in younger children, compounded by ongoing assessments at school. Therefore, the additional academic stress could exacerbate any nutritional effects on cognitive function ${ }^{(11)}$.
However, only three studies to date have examined the effect of the GI of breakfast on cognitive function in an adolescent population ${ }^{(13-15)}$. Of these studies, one has shown that a high-GI glucose drink and breakfast omission resulted in a decline in attention and memory during the school morning, but this decline was reduced following the consumption of low-GI breakfast cereals ${ }^{(13)}$. However, nutritional information on the breakfasts was not provided and there was a wide age range of participants (9-16-year-olds), not all of whom were adolescents. In contrast, another study has shown that $90 \mathrm{~min}$ after breakfast consumption, 14-17-yearolds were able to remember more items following a high-GI breakfast compared with a low-GI breakfast ${ }^{(15)}$. It has been suggested that this enhanced memory could be the result of higher blood glucose concentrations following the high-GI breakfast, which would be beneficial under the conditions of divided attention. However, there were no significant differences in blood glucose concentration between the trials and only one element of cognitive function was assessed, namely verbal episodic memory. In the one further study, it

Abbreviations: GI, glycaemic index; IAUC, incremental area under the curve.

*Corresponding author: S. B. Cooper, fax +44 1509 226301, email s.b.cooper@lboro.ac.uk 
has been reported that performance on a speed of information processing task and a serial sevens task was enhanced following a low-GI breakfast, whereas a high-GI breakfast was beneficial for immediate word recall ${ }^{(14)}$. However, the breakfasts provided were not matched on key variables, such as energy and carbohydrate content.

Thus, the findings are equivocal regarding the effects of the GI of breakfast on cognitive function in adolescents, with the possibility that the effects of high- and low-GI breakfasts vary for different elements of cognitive function. Therefore, the aim of the present study was to conduct a randomised controlled trial, using a cross-over design, to assess the effects of a high-GI breakfast, a low-GI breakfast and breakfast omission on cognitive function in adolescent school children. The study employed a battery of computer tests to assess various elements of cognitive function. Furthermore, blood glucose and plasma insulin concentrations were measured to allow a possible insight into the mechanisms for any effects of the GI of breakfast on cognitive function in adolescent school children.

\section{Methodology}

\section{Study design}

The study was conducted according to the guidelines laid down in the Declaration of Helsinki and all procedures were approved by Loughborough University Ethical Advisory Committee. Participants were recruited from two local schools, and in accordance with the ethical guidelines of the British Education Research Authority for school-based research, school-level consent was obtained from head teachers. In addition, written parental informed consent was obtained and a health screen questionnaire completed to ensure all participants were in good health.

Each participant undertook a familiarisation session followed by three experimental trials. During familiarisation, which preceded the first experimental trial by $7 \mathrm{~d}$, the protocol of the study was explained to participants and they were provided with an opportunity to familiarise themselves with the methods involved. Participants were allowed to repeat the cognitive function tests until they felt comfortable with them, to negate any potential learning effects.

The study employed a randomised cross-over design and was order balanced, with participants blind until arrival at school on each day of testing. The experimental trials consisted of a high-GI breakfast trial, a low-GI breakfast trial and breakfast omission trial (where breakfast was provided upon completion of the protocol). Therefore, participants acted as their own controls and the effects of the different breakfast conditions can be assessed as within-subject factors, yielding greater sensitivity. Trials were scheduled $7 \mathrm{~d}$ apart and participants reported to school at the normal time. The experimental protocol is shown in Fig. 1.

Upon arrival at school, participants rested in a seated position for $10 \mathrm{~min}$ and then a capillary blood sample was taken. The protocol commenced as participants began breakfast on the high- and low-GI trials, whereas on the breakfast omission trial, the protocol commenced after the resting capillary blood sample had been collected. On the high- and lowGI trials, participants were given 15 min to consume breakfast, whereas on the breakfast omission trial, participants rested for $15 \mathrm{~min}$. Capillary blood samples and the cognitive function tests were completed during the subsequent monitoring period. A $120 \mathrm{~min}$ monitoring period was selected based upon recommendations which suggest that this is a sufficient period of time to elicit the different glycaemic responses between the meals ${ }^{(16)}$. This is also the period of time after which it is suggested that the effects of breakfast consumption on cognitive function will become apparent in young people p $^{(5,13,14,17)}$

\section{Participants}

A total of fifty-two participants aged 12-14 years were recruited to participate in the study. However, eleven participants were removed from the study because they were absent from school for one or more of the experimental trials ( $n 9$ ), or did not follow the dietary requirements $(n 2)$. Therefore, forty-one participants completed the study. During familiarisation, height, body mass and waist circumference were measured. Height was measured using a Leicester Height Measure (Seca, Hamburg, Germany), accurate to $0 \cdot 1 \mathrm{~cm}$. Body mass was measured using a Seca 770 digital scale (Seca, Hamburg, Germany), accurate to $0 \cdot 1 \mathrm{~kg}$. These measures allowed the determination of BMI, calculated by dividing body mass $(\mathrm{kg})$ by the square of the height $\left(\mathrm{m}^{2}\right)$. Waist circumference was measured at the narrowest point of the torso between the xiphoid process of the sternum and the iliac crest, to the nearest $0 \cdot 1 \mathrm{~cm}$. Table 1 provides the physiological characteristics of the participants.

\section{Dietary control}

Participants were asked to consume a meal of their choice the evening before their first experimental trial and then to

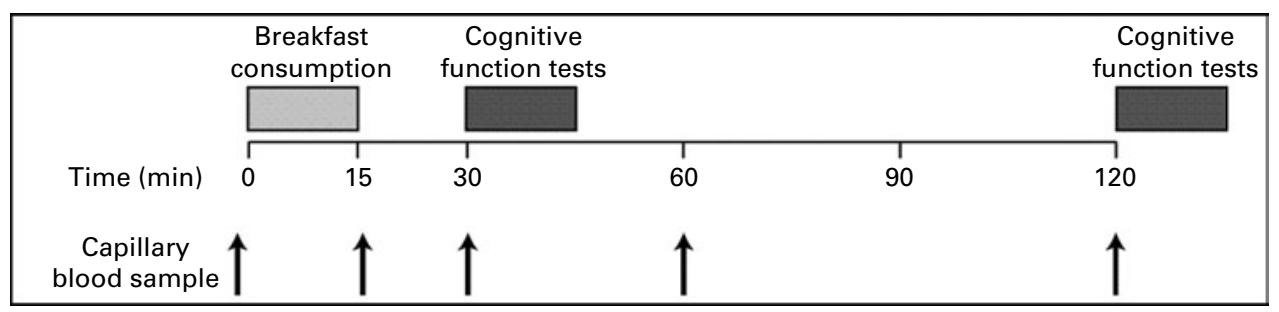

Fig. 1. Experimental protocol. 
repeat this meal for each of the subsequent trials. Participants fasted from 22.00 hours the evening before each experimental trial. In order to maintain euhydration, participants were allowed to drink water ad libitum during this time. In addition, participants avoided any unusually vigorous exercise for $24 \mathrm{~h}$ before each experimental trial. Before each main trial, a telephone call was made to participants to remind them of this information. On the day of each experimental trial, participants were asked to indicate if they had followed the above requirements when they arrived at school.

\section{Capillary blood samples}

Capillary blood samples were taken at baseline and at 15, 30, 60 and $120 \mathrm{~min}$ after breakfast consumption on each trial. Capillary blood samples were preferred to venous blood samples in the present study because they are more sensitive to glycaemic responses and show a lower between-subject variation $^{(16,18-20)}$. Furthermore, capillary blood samples were more acceptable to the adolescents participating.

Participants' hands were warmed via submersion in warm water to increase capillary blood flow. A Unistik single-use lancet (Unistik Extra, 21 gauge, $2 \cdot 0 \mathrm{~mm}$ depth; Owen Mumford Limited, Oxford, UK) was used and the blood collected into two $300 \mu \mathrm{l}$ EDTA-coated microvettes (Sarstedt Limited, Leicester, UK). Then, two $25 \mu \mathrm{l}$ whole blood samples were removed using $25 \mu \mathrm{l}$ plain pre-calibrated glass pipettes (Hawksley Limited, Lancing, West Sussex, UK), immediately deproteinised in $250 \mu \mathrm{l}$ of $2.5 \%$ ice-cold perchloric acid in $1.5 \mathrm{ml}$ plastic vials and centrifuged at $7000 \mathrm{rpm}$ for $4 \mathrm{~min}$ (Eppendorph 5415C; Eppendorph Limited, Hamburg, Germany). The remaining whole blood was also centrifuged at $7000 \mathrm{rpm}$ for $4 \mathrm{~min}$ (Eppendorph 5415C; Hamburg, Germany) and the plasma removed and placed into $500 \mu \mathrm{l}$ plastic vials. All samples were frozen at $-20{ }^{\circ} \mathrm{C}$ until analysis.

Blood glucose concentrations were determined using a commercially available kit (GOD-PAP method, GL 2610; Randox Laboratories Limited, Crumlin, Co. Antrim, UK) and were analysed spectrophotometrically (Cecil CE393 digital grating spectrophotometer; Cecil Instruments, Cambridge, UK). Plasma insulin concentrations were determined using an ELISA (Mercodia Limited, Uppsala, Sweden). Incremental area under the curve (IAUC) for blood glucose and plasma insulin was calculated using previously described methods ${ }^{(21)}$.

\section{Cognitive function tests}

The battery of cognitive function tests was administered via a laptop computer and took approximately $15 \mathrm{~min}$ to complete. The battery of tests included a Stroop test, the Sternberg paradigm and a Flanker task. Written instructions appeared on the screen at the start of each test, which were repeated verbally by an investigator. Each cognitive function test was preceded by three to six practice stimuli, where feedback was provided regarding whether the participants' response was correct or not. This allowed the participants to re-familiarise themselves with each of the tests and ensure that instructions were 
fully understood. Results from these practice stimuli were discarded and once the test started no feedback was provided. The cognitive function tests were found to be suitable (avoiding floor or ceiling effects) for the study population during familiarisation and were administered in the following order.

Stroop test. The Stroop test measures the sensitivity to interference and the ability to suppress an automated response and is a widely used measure of executive function $^{(22,23)}$. The Stroop test consisted of two levels (baseline and complex). Both levels involved a test word being placed in the centre of the screen, with the target and distractor presented randomly on the right or left of the test word. The target position was counterbalanced for the left and right side within each level of the test. The participant was asked to respond as quickly as possible, using the left and right arrow keys, to identify the position of the target word.

The baseline level contained twenty stimuli, where the test word was printed in white and the participant had to select the target word, from the target and distractor, which were also printed in white. The colour-interference level contained forty stimuli and involved the participant selecting the colour the test word was written in, rather than the actual word (which was an incongruent colour), again using the right and left arrow keys to identify the target. The choices remained on the screen until the participant responded. The variables of interest were the response times of correct responses and the proportion of correct responses made.

Sternberg paradigm. The Sternberg paradigm $^{(24)}$ is a test of working memory and has three levels. Each level used a different working memory load; one, three or five items. On the baseline (number) level, the target was always the number ' 3 '. This level contained sixteen stimuli and provides a measure of basic information processing speed. The threeand five-item levels had target lists of three and five letters, respectively, each containing thirty-two stimuli.

At the start of each level, the target items were displayed together with instructions to press the right arrow key if the stimulus was a target item and the left arrow key otherwise. The correct responses were counterbalanced on each level between the right and left arrow keys. The choice stimuli were presented on the centre of the screen with an inter-stimulus interval of $1 \mathrm{~s}$, during which the screen was blank. The choices remained on the screen until the participant responded. The variables of interest were the response times of correct responses and the proportion of correct responses made.

Flanker task. The Flanker task assesses aspects of attention and has two levels, congruent and incongruent. On the congruent level, five arrows appear on the screen, all pointing in the same direction (left or right). The participant is asked to select the arrow key pointing in the same direction as the arrows. On the incongruent level, the arrows point in different directions and the participant selected the arrow key pointing in the same direction as the central arrow. On both levels, the arrows were presented in green on a black background, after a varied delay of $400-4000 \mathrm{~ms}$. The items remained on the screen until the participant responded. The variables of interest were the response times of correct responses and the proportion of correct responses made.

\section{Breakfast}

Breakfast was provided after the resting measures had been taken and participants had $15 \mathrm{~min}$ to consume breakfast. The high- and low-GI breakfasts both contained $1.5 \mathrm{~g} / \mathrm{kg}$ body mass available carbohydrate and were matched for energy, protein and fat content. Water was provided at the start of the protocol on the high-GI $(150 \mathrm{ml})$ and breakfast omission $(350 \mathrm{ml})$ trials, to ensure that total water intake was the same between the trials. Furthermore, $150 \mathrm{ml}$ of water were provided after $60 \mathrm{~min}$ on each trial. The breakfast composition for a $50 \mathrm{~kg}$ participant is shown in Table 2 .

\section{Statistical analysis}

The blood glucose and plasma insulin data were analysed using SPSS (version 16; SPSS Inc., Chicago, IL, USA) via twoway ANOVA for repeated measures (trial $\times$ session time).

The cognitive function data were analysed using $R$ (www.r-project.org, version 2.9.1). Linear mixed-effects models were used to analyse the data, corrected for repeated measures with a random effect for each participant. Response time analyses were performed using the nlme package and accuracy analyses were performed with the lme 4 package with a binomial outcome data distribution to properly account for the binomial (correct/incorrect) accuracy scores. All analyses were conducted using a three-way trial $\times$ session time $\times$ test level interaction. Where the three-way interaction was not significant, a two-way trial $\times$ session time interaction was conducted. For all analyses, significance was set as $P<0 \cdot 05$.

Table 2. Composition of high-glycaemic index (HGl) and low-Gl (LGI) breakfasts for a $50 \mathrm{~kg}$ participant

\begin{tabular}{|c|c|c|}
\hline Breakfasts & $\mathrm{HGI}$ & LGI \\
\hline \multicolumn{3}{|l|}{ Food items (g) } \\
\hline Cornflakes* & 55 & \\
\hline White bread $\dagger$ & 42 & \\
\hline Margarine $\ddagger$ & 6 & \\
\hline $1 \%$ Fat milk§ & 216 & 217 \\
\hline Muesli|| & & 75 \\
\hline Appleף & & 150 \\
\hline \multicolumn{3}{|l|}{ Macronutrients } \\
\hline $\mathrm{E}(\mathrm{kJ})$ & $1765 \cdot 6$ & $1757 \cdot 3$ \\
\hline $\mathrm{CHO}(\mathrm{g})$ & $75 \cdot 0$ & $75 \cdot 0$ \\
\hline Fat $(\mathrm{g})$ & $7 \cdot 2$ & 6.4 \\
\hline Protein (g) & $14 \cdot 3$ & $15 \cdot 5$ \\
\hline $\mathrm{Gl}^{* *}$ & 72 & 48 \\
\hline Glycaemic load†† & 54 & 36 \\
\hline \multicolumn{3}{|c|}{ 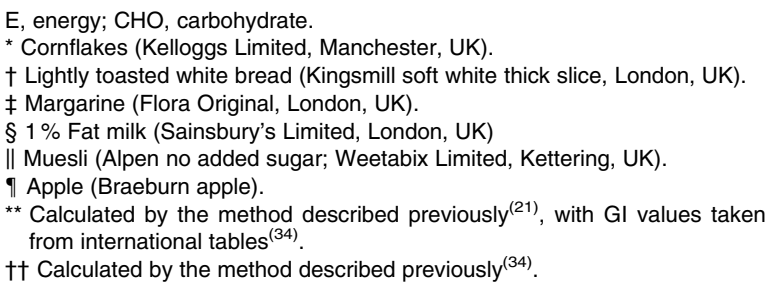 } \\
\hline
\end{tabular}




\section{Results}

\section{Cognitive function tests}

All participants completed all cognitive function tests at each time point ( $n 41$ for all analysis). For all timed cognitive tests, the response times were first log-transformed to normalise the distributions, which exhibited the right-hand skew typical of human response times. Minimum response time cut-offs were then chosen based on what may reasonably be expected to be the fastest possible human response to the given stimuli (200-300 ms, depending on task complexity) to exclude unreasonably fast responses, which relate to response key presses before stimuli have even been perceived. Maximum response time cut-offs were determined so as to remove unreasonably long right-hand tails for a normal distribution, corresponding to $5 \mathrm{SD}$ individually for each test and test level. Stroop test

Response times. Only response times of correct responses were used for analysis. Using the methods previously described, responses faster than $250 \mathrm{~ms}$ for both test levels and slower than $2500 \mathrm{~ms}$ for the baseline level and $4000 \mathrm{~ms}$ for the complex level were removed.

Response times were quicker following the high-GI breakfast when compared with the low-GI breakfast (main effect of trial, $t(1,13537)=2 \cdot 1, P=0 \cdot 031)$. Response times following the high-GI breakfast tended to be quicker 120 min following breakfast consumption when compared with breakfast omission, an effect specific to the complex level, but this did not reach statistical significance (trial $\times$ session time $\times$ test level interaction, $t(1,13530)=1 \cdot 8, P=0.079$; Fig. 2). Furthermore, response times following the low-GI breakfast were quicker 120 min following breakfast consumption when compared with breakfast omission, and, again, this effect was specific to the complex level (trial $\times$ session time $\times$ test level interaction, $t(1,9019)=2 \cdot 6, P=0 \cdot 009$; Fig. 2). However, the pattern of change in response times across the morning between the high- and low-GI trials was not different $($ trial $\times$ session time $\times$ test level interaction and trial $\times$ time interaction, both $P>0 \cdot 05)$.

Accuracy. Students achieved more correct responses following the low-GI breakfast compared with following both the high-GI breakfast (main effect of trial, effect size = $0 \cdot 011, z(1,14820)=2 \cdot 1, P=0 \cdot 039)$ and breakfast omission (main effect of trial, effect size $=0.274, z(1,14820)=3 \cdot 6$, $P<0.001)$. However, there was no significant difference in the proportion of correct responses between the high-GI and breakfast omission trials (main effect of trial, $P=0 \cdot 150$ ).

On the high-GI trial, there was a greater decrease in accuracy across the morning when compared with the low-GI trial (trial $\times$ session time interaction, effect size $=0.024$
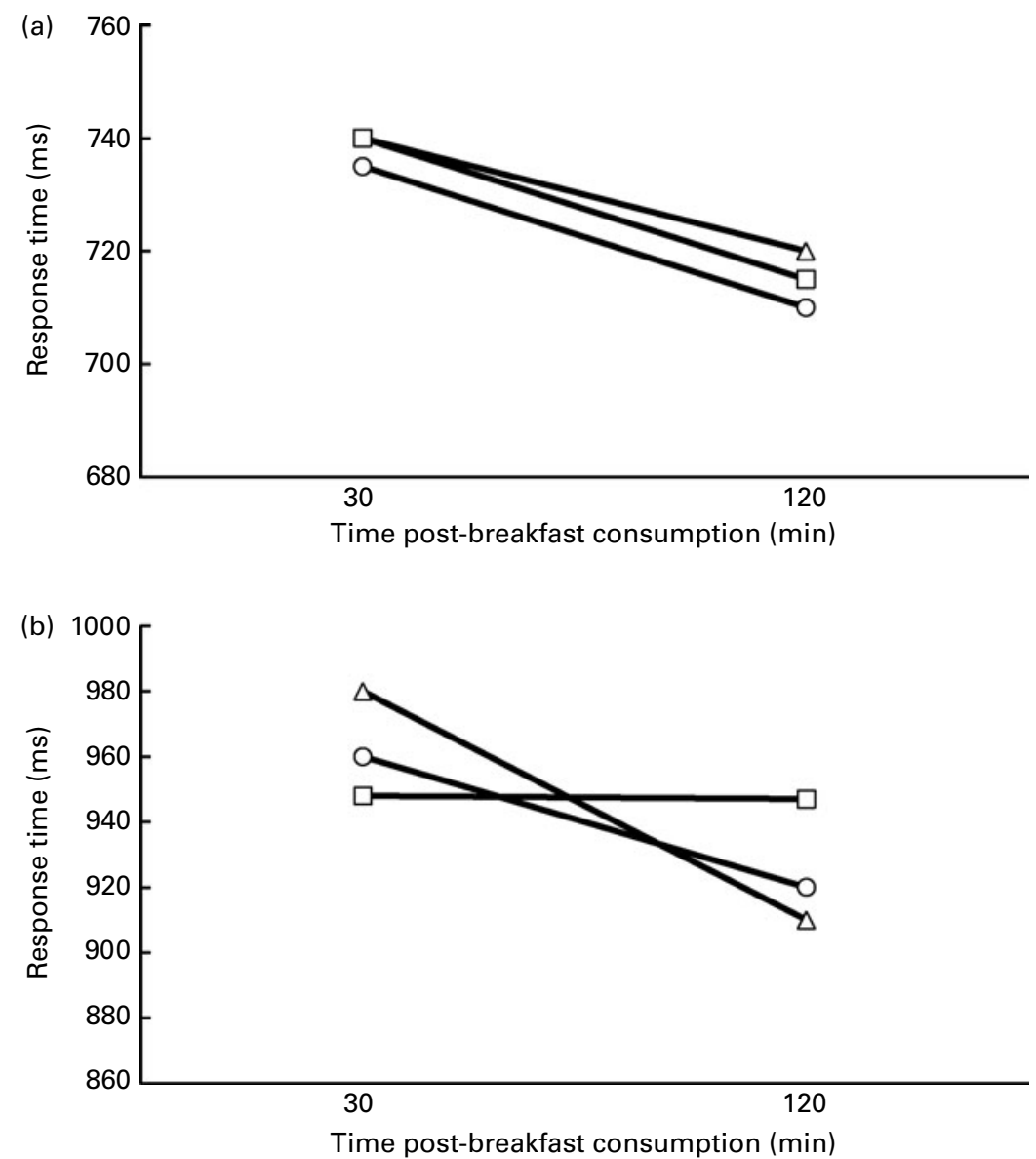

Fig. 2. Response times across the high-glycaemic index (HGI, $-\mathrm{O}-$ ), low-GI (LGI, $-\checkmark)$ ) and breakfast omission (NBF, $-\square$ ) trials on the (a) baseline and (b) complex levels of the Stroop test $(n 41)$. LGI $v$. NBF: $P=0.009$; trial $\times$ session time $\times$ test level interaction. 


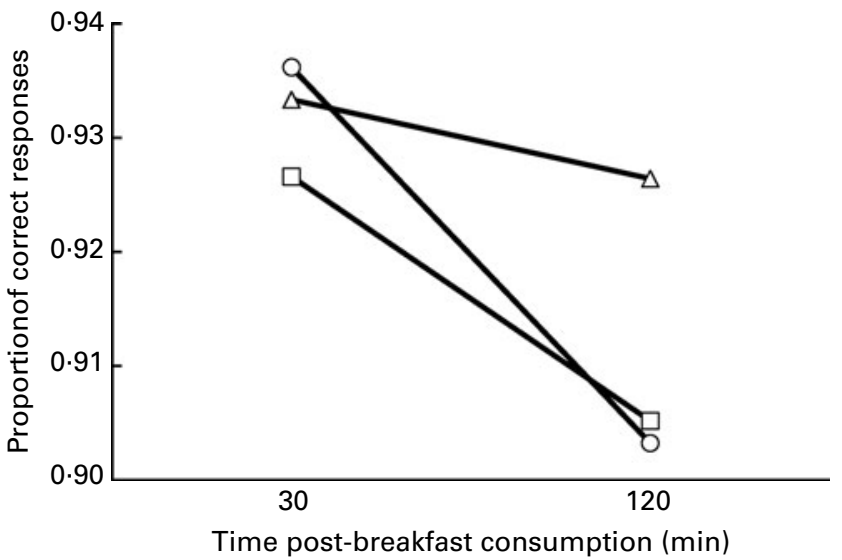

Fig. 3. Accuracy across the morning on the high-glycaemic index ( $\mathrm{HGI},-\mathrm{O}-)$, low-GI (LGI, $-\triangle)$ ) and breakfast omission ( $\square-$ ) trials on the Stroop test $(n 41)$. LGI $v$. HGI: $P=0.033$; trial $\times$ session time interaction.

$z(1,14820)=2 \cdot 1, P=0 \cdot 033$; Fig. 3). However, this effect was not specific to the test level (trial $\times$ session time $\times$ test level interaction, $P=0 \cdot 121)$. There were no other significant interactions between the different conditions and the testing time and/or the test level (all $P>0 \cdot 05$ ).

Sternberg paradigm

Response times. Only response times of correct responses were used for analysis. Using the methods previously described, responses faster than $200 \mathrm{~ms}$ and slower than $2000 \mathrm{~ms}$ for all test levels were removed.

Overall, participants responded quicker following breakfast omission compared with following both the high-GI breakfast (main effect of trial, $t(1,17468)=3.6, P<0.001)$ and the lowGI breakfast (main effect of trial, $t(1,17468)=2 \cdot 5, P=0 \cdot 011$ ). However, while response times remained similar across the morning following the high-GI breakfast, there was a greater improvement in response times across the morning following the low-GI breakfast (trial $\times$ session time interaction, $t(1$, $17438)=2 \cdot 5, P=0 \cdot 013$; Fig. 4)

Accuracy. Overall, participants achieved a greater proportion of correct responses following the low-GI breakfast when compared with breakfast omission (main effect of trial, effect size $=0 \cdot 010, z(1,19520)=2 \cdot 1, \quad P=0 \cdot 036)$, but

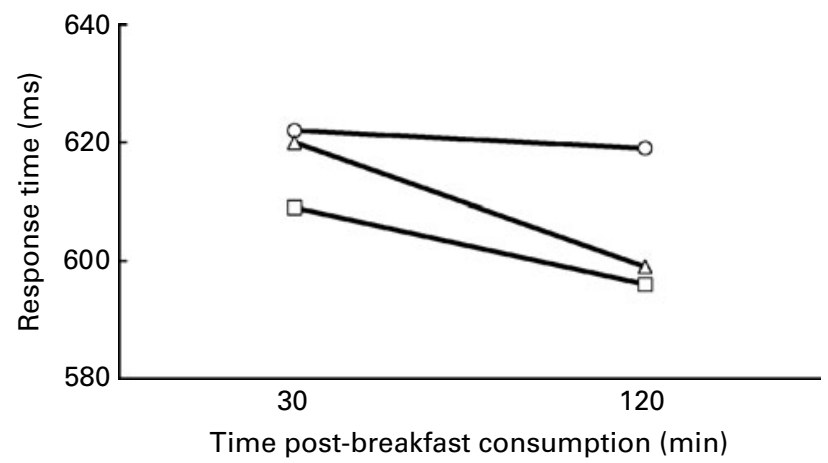

Fig. 4. Response times across the morning on the high-glycaemic index ( $\mathrm{HGl},-\mathrm{O})$ ), low-GI ( $\mathrm{LGl},-\triangle)$ ) and breakfast omission (- $\square$-) trials on the Sternberg paradigm ( $n 41)$. LGI $v$. HGI: $P=0.013$; trial $\times$ session time interaction. there was no difference between the low-GI and high-GI or the high-GI and breakfast omission trials (main effects of trial, $P=0 \cdot 118$ and $P=0.586$, respectively)

While accuracy was similar across the morning between the trials on the easier levels (Fig. 5(a) and (b)), on the more complex levels of the Sternberg paradigm, accuracy was better maintained across the morning following the low-GI breakfast when compared with the high-GI breakfast $($ trial $\times$ session time $\times$ test level interaction, effect

(a)

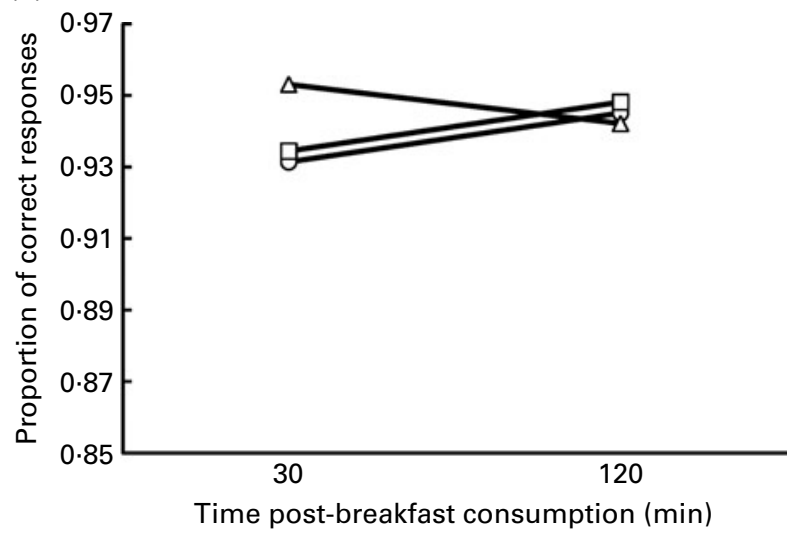

(b)

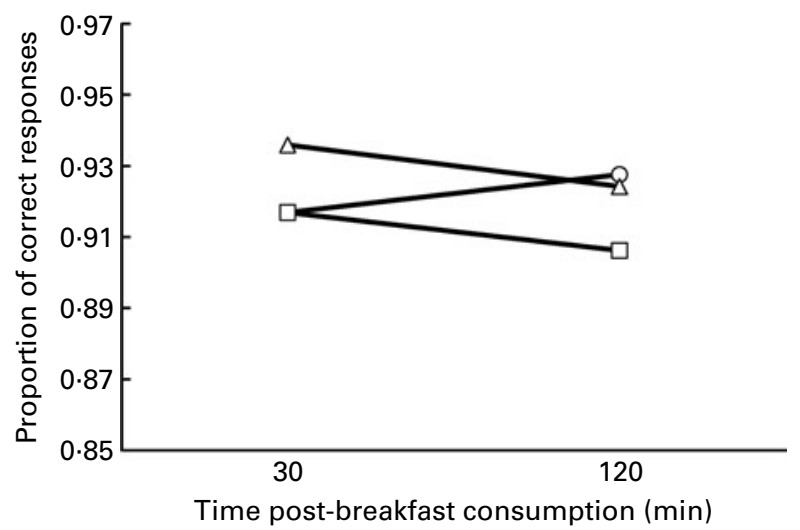

(c)

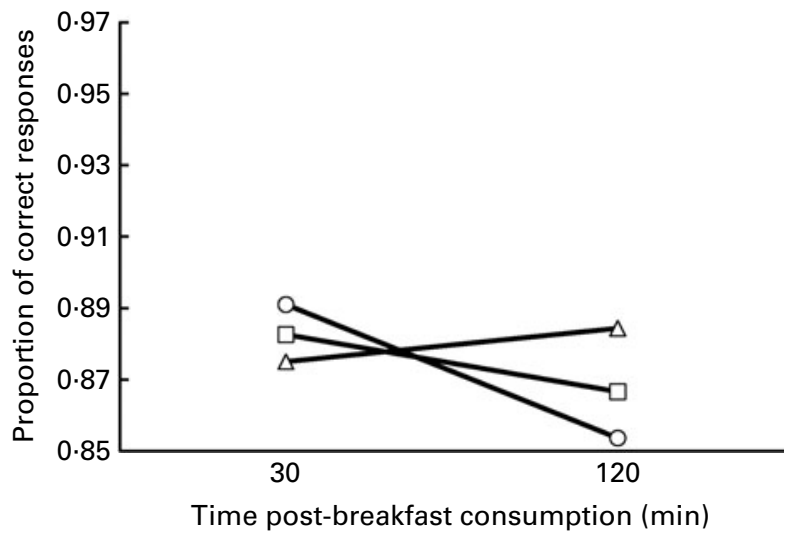

Fig. 5. Accuracy across the morning on the (a) number, (b) three-letter and (c) five-letter levels of the Sternberg paradigm on the high-glycaemic index (HGI, -O), low-GI (LGI, $-\triangle$ ) and breakfast omission (NBF, $-\square$ ) trials ( $n$ 41). LGI $v$. HGI: $P=0.002$; trial $\times$ session time $\times$ test level interaction. LGI $v$. NBF: $P=0.051$; trial $\times$ session time $\times$ test level interaction. 


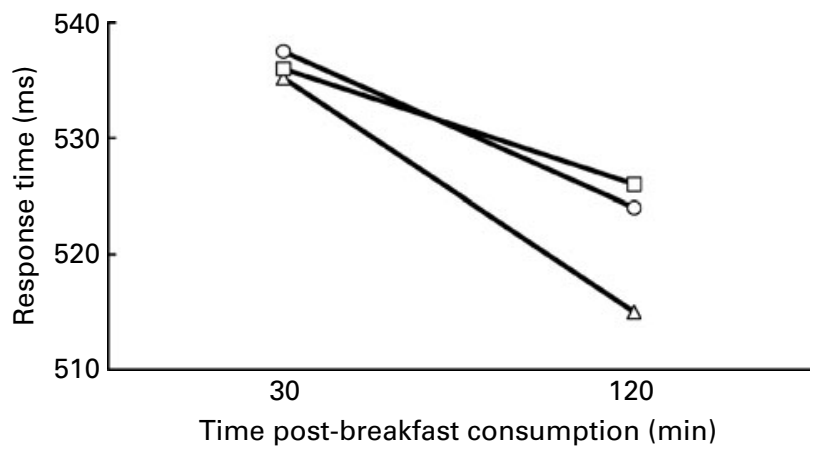

Fig. 6. Response times across the morning on the high-glycaemic index ( $\mathrm{HGl},-\mathrm{O}-$ ), low-Gl ( $\mathrm{LGl},-\triangle$ ) and breakfast omission (NBF, $-\square-$ ) trials on the Flanker task ( $n 41)$. LGI $v$. NBF: $P=0.045$; trial $\times$ session time interaction.

size $=0 \cdot 040, z(1,19520)=3 \cdot 1, P=0 \cdot 002 ;$ Fig. 5(c)). There was also a tendency for accuracy to be better maintained across the morning following the low-GI breakfast when compared with the breakfast omission trial; again, this effect was only evident on the more complex levels (trial $\times$ session time $\times$ test level interaction, effect size $=0.025$, $z(1,19520)=2 \cdot 0, P=0.051$; Fig. 5(c)).

(a)

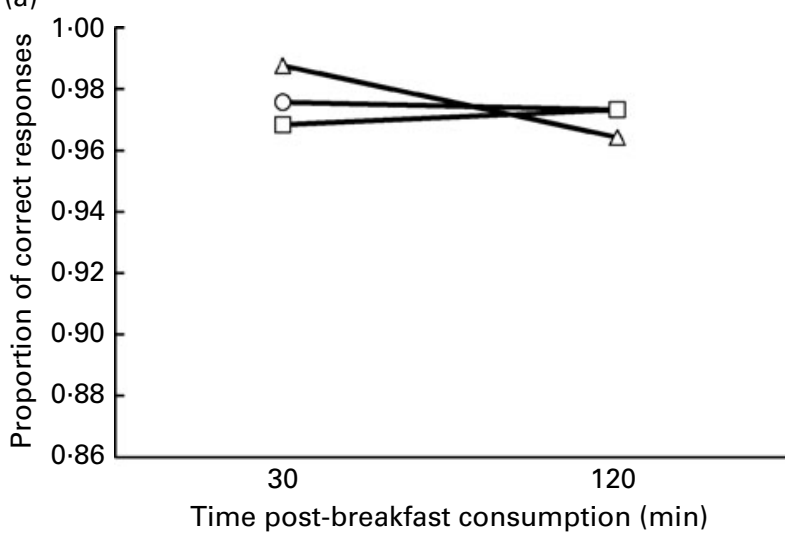

(b)

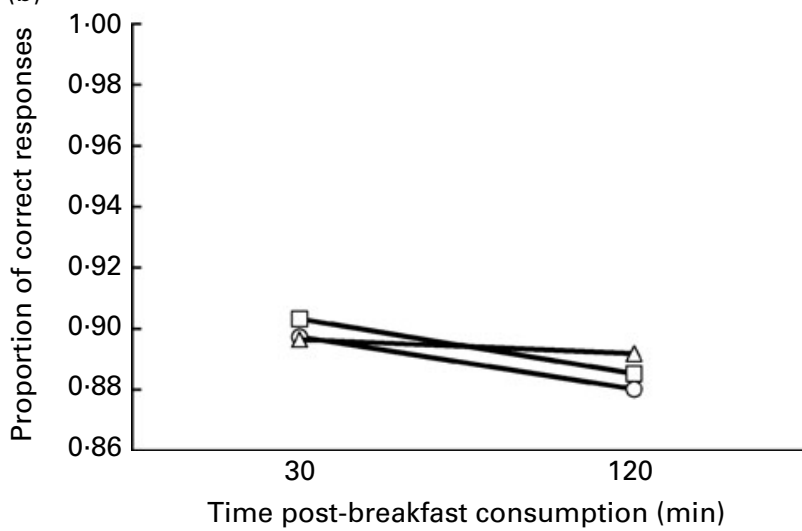

Fig. 7. Accuracy across the morning on the (a) congruent and (b) incongruent levels of the Flanker task on the high-glycaemic index ( $\mathrm{HGl},-\mathrm{O}-)$, low-GI (LGI, $-\checkmark$ ) and breakfast omission (NBF, $\square-$ ) trials ( $n$ 41). LGI v. HGI: $P=0.014$; trial $\times$ session time $\times$ test level interaction. LGI NBF: $P=0.001$; trial $\times$ session time $\times$ test level interaction.

\section{Flanker task}

Response times. Only response times of correct responses were used for analysis. Using the methods previously described, responses faster than $100 \mathrm{~ms}$ and slower than $2500 \mathrm{~ms}$ were removed.

Overall, response times between the trials were not significantly different (main effects of trial: high-GI $v$. low-GI, $P=0.497$; high-GI $v$. breakfast omission, $P=0 \cdot 909$ ); low-GI $v$. breakfast omission, $P=0.634)$. There was a greater improvement in response times across the morning following the low-GI breakfast when compared with breakfast omission $($ trial $\times$ session time interaction, $t(1,13630)=2 \cdot 0, P=0 \cdot 045$; Fig. 6). Apart from this trial $\times$ session time interaction, response times across the morning were similar between the trials on both test levels (all other interactions, $P>0 \cdot 05$ ).

Accuracy. Overall, there was no significant difference in the proportion of correct responses between the trials (main effects of trial: high GI $v$. low-GI, $P=0.931$; high GI $v$. breakfast omission, $P=0.859$; low-GI $v$. breakfast omission, $P=0.805$ ). However, on the incongruent (more complex) level, accuracy was better maintained across the morning following the low-GI breakfast compared with the high-GI breakfast (trial $\times$ session time $\times$ test level interaction, effect size $=0.033, \quad z(1, \quad 14700)=2.5, \quad P=0.014 ; \quad$ Fig. $7(\mathrm{~b}))$ and breakfast omission (trial $\times$ session time $\times$ test level interaction, effect size $=0.042, z(1,14700), P=0.001 ;$ Fig. $7(b))$.

\section{Capillary blood samples}

Blood glucose. Blood glucose concentrations and the pattern of response across the morning were different between the trials (main effect of trial, time and trial $\times$ time interaction, all $P<0.001)$. However, because the differences between breakfast omission and breakfast consumption were expected, the following results consider only the high-GI and low-GI trials, with the breakfast omission trial shown in the figures only for illustration purposes.

Blood glucose concentration was significantly higher on the high-GI trial, compared with the low-GI trial (main effect of

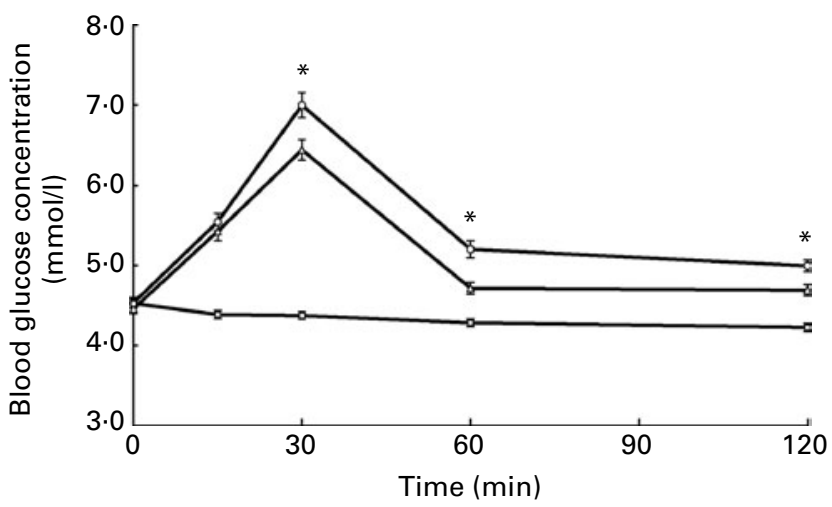

Fig. 8. Blood glucose concentrations across the high-glycaemic index (HGI, -O) ), low-GI (LGI, $-\triangle$ ) and breakfast omission ( $-\square-)$ trials. Values are means, with their standard errors represented by vertical bars $(n 41)$. LGI $v$. HGl: $P<0.001$; trial $\times$ time interaction. ${ }^{*}$ Mean values were significantly different for HGI $v$. LGI $(P<0.001)$. 


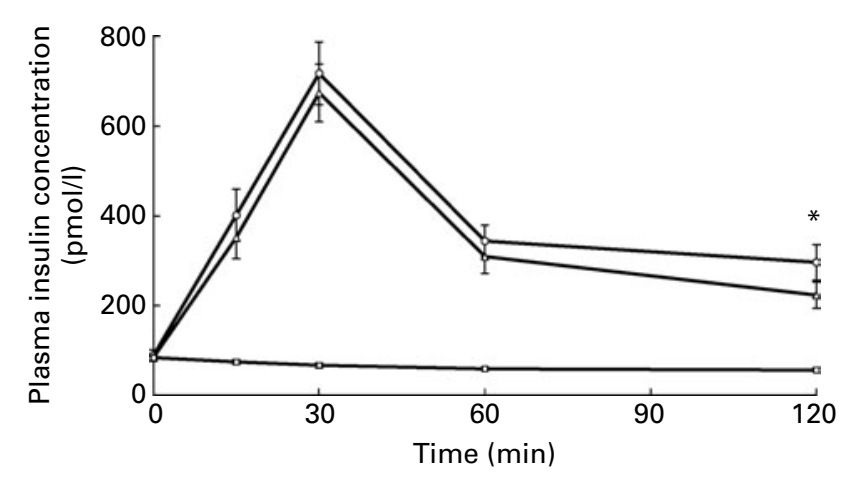

Fig. 9. Plasma insulin concentrations across the high-glycaemic index (HGI, -O), low-GI (LGI, - ) ) and breakfast omission ( $\square$ ) trials. Values are means, with their standard errors represented by vertical bars ( $n 41)$. LGI $v$. HGI: $P=0.507$; trial $\times$ time interaction. * Mean values were significantly different for HGI $v$. LGI $(P=0.008)$.

trial, $F(1,40)=44 \cdot 4, P<0 \cdot 001)$. As expected, blood glucose concentrations increased after both the high- and low-GI breakfasts, peaking at $30 \mathrm{~min}$, before returning towards resting concentrations (main effect of time, $F(4,160)=138 \cdot 3$, $P<0 \cdot 001)$. However, blood glucose concentrations reached a higher peak at $30 \mathrm{~min}$ on the high-GI trial compared with the low-GI trial $(7.01 \quad v .6 .46 \mathrm{mmol} / \mathrm{l}$, respectively) and remained higher 60 and $120 \mathrm{~min}$ following breakfast $(60 \mathrm{~min}$ : 5.23 v. $4.71 \mathrm{mmol} / \mathrm{l}$; $120 \mathrm{~min}: 5.01 \quad$ v. $4.69 \mathrm{mmol} / \mathrm{l}$, both $P<0 \cdot 001)$. This produced a significant interaction between trial and time (trial $\times$ time interaction, $F(4,160)=5.9$, $P<0 \cdot 001$; Fig. 8). Furthermore, blood glucose IAUC was also greater following the high-GI breakfast compared with following the low-GI breakfast $(116.6$ v. $80.9 \mathrm{mmol} / 1 \times 120 \mathrm{~min}$, respectively, $P<0 \cdot 001$ ).

Plasma insulin. Plasma insulin concentrations and the pattern of response across the morning were different between the trials (main effect of trial, time and a trial $\times$ time interaction, all $P<0 \cdot 001)$. However, because the differences between breakfast omission and breakfast consumption were expected, the following results consider only the highGI and low-GI trials, with the breakfast omission trial shown in the figures only for illustration purposes.

Plasma insulin concentration was significantly higher on the high-GI trial, compared with the low-GI trial (main effect of trial, $F(1,40)=4.3, P=0.045)$. As expected, plasma insulin concentrations increased after both the high- and low-GI breakfasts, peaking at $30 \mathrm{~min}$, before returning towards resting concentrations (main effect of time, $F(4,160)=68 \cdot 1$, $P<0 \cdot 001)$. However, there was no difference in the pattern of change in plasma insulin concentration across the morning between the high- and low-GI trials (trial $\times$ time interaction, $F(4,160)=0.5, P=0.507$; Fig. 9), nor was there a difference in plasma insulin IAUC following the high- and low-GI breakfasts $(36590 v .31651 \mathrm{pmol} / 1 \times 120 \mathrm{~min}$, respectively, $P=0 \cdot 063)$.

\section{Discussion}

The main finding of the present study is that a low-GI breakfast enhanced cognitive function in adolescents, when compared with both a high-GI breakfast and breakfast omission.
Across all three cognitive function tests (Stroop test, Sternberg paradigm and Flanker task), a low-GI breakfast enhanced both response times and accuracy later in the morning when compared with a high-GI breakfast, breakfast omission or both, particularly on the more cognitively demanding levels of the tests employed. Ingestion of the low-GI breakfast also resulted in a lower peak blood glucose concentration when compared with a high-GI breakfast, produced a smaller overall glycaemic response and tended to produce a smaller overall insulinaemic response.

\section{Stroop test}

Response times on the Stroop test were quicker overall on the high-GI trial, when compared with the low-GI trial. Furthermore, on the incongruent (more complex) level of the Stroop test, there was a greater improvement in response times across the morning following a low-GI breakfast when compared with breakfast omission (Fig. 2(b)). Accuracy was also better maintained across the morning following a lowGI breakfast when compared with a high-GI breakfast (Fig. 3).

Another study that examined the effects of the GI of breakfast on the performance on the Stroop test has reported that a high-GI breakfast was more beneficial than a low-GI breakfast, but only in the group that had consumed a high-glycaemic load breakfast ${ }^{(25)}$. It has also been reported that neither the GI nor the glycaemic load of breakfast affects adolescents' performance on the Stroop test ${ }^{(14)}$. However, the earlier studies suffered from a number of methodological weaknesses, including providing high- and low-glycaemic load meals that were not matched for energy content ${ }^{(14,25)}$, not reporting whether response times and/or accuracy were assessed on the Stroop test ${ }^{(25)}$ and furthermore, not employing a cross-over design, with participants consuming only the high- or low-glycaemic load breakfasts ${ }^{(14,25)}$. In comparison, in the present study, participants performed all trials in a randomised cross-over design and the breakfasts were matched on key variables such as energy and carbohydrate content.

It has previously been suggested that higher blood glucose concentrations are associated with better performance on the Stroop test ${ }^{(26)}$. However, studies from which this conclusion was drawn focused on a nutritional intervention (e.g. breakfast or lunch provision) $v$. continued fasting ${ }^{(1)}$. Similarly, in our previous work, we have found that breakfast consumption (which was associated with higher blood glucose concentrations) improved performance on the Stroop test compared with breakfast omission ${ }^{(5)}$. However, the present study compares two nutritional interventions (high- and low-GI breakfasts) with breakfast omission, extending the previous work that examined nutritional interventions $v$. fasting.

Thus, while the enhanced performance following both the high- and low-GI breakfasts, compared with following breakfast omission, may be mediated by the higher blood glucose concentrations $^{(26)}$, when comparing the high- and low-GI trials, it seems that the higher blood glucose concentrations enhance response times, but they are to the detriment of accuracy (possibly causing a speed-accuracy trade-off). Alternatively, blood glucose concentrations (within the 
postprandial ranges following the high- and low-GI breakfasts) may not be the only determinant of performance on the Stroop test, and other factors such as improved insulin sensitivity following a low-GI breakfast ${ }^{(27)}$ may also play a role in determining performance. However, the present study does not allow us to provide details of the mechanistic pathways determining cognitive performance, rather it only allows us to speculate on such mechanisms.

\section{Sternberg paradigm}

There was a greater improvement in response times across the morning on the Sternberg paradigm, a test of working memory, following a low-GI breakfast compared with a high-GI breakfast (Fig. 4). Furthermore, accuracy was better maintained across the morning following a low-GI breakfast compared with following both the high-GI breakfast and breakfast omission trials, but this was only evident on the more complex level (Fig. 5(c)).

The findings of the present study are consistent with an earlier study in 9-16-year-olds, which showed a greater improvement in response times across the morning following a low-GI breakfast, compared with both a high-GI breakfast and breakfast omission, but in the earlier study, the accuracy of working memory was not investigated ${ }^{(13)}$. The accuracy of working memory has been examined previously in children, with 9-11-year-old females showing an enhanced accuracy following a low-GI breakfast as opposed to a high-GI breakfast. However, there were no effects of the different breakfasts in the 9-11-year-old males or in 6-8-year-old males or females ${ }^{(28)}$. Thus, the present study is the first to examine the effects of the GI of breakfast on both the speed and accuracy of working memory in an adolescent population.

It has been previously suggested that the improvement in working memory following a low-GI breakfast could be due to better maintenance of blood glucose concentrations after a 'simulated' low-GI breakfast ${ }^{(10)}$. However, in the present study, following the 'real-life' low-GI breakfast, blood glucose concentrations were lower than following the high-GI breakfast, thus contradicting the suggestion that maintenance of higher blood glucose concentrations within normal postprandial ranges is a key determinant of working memory performance.

\section{Flanker task}

There was a greater improvement in response times across the morning on the Flanker task, which is a test of selective attention, following a low-GI breakfast when compared with breakfast omission (Fig. 6). Furthermore, accuracy on the incongruent (more difficult) level was better maintained across the morning following a low-GI breakfast when compared with both the high-GI breakfast and breakfast omission (Fig. 7(b)).

The improvement in accuracy on the Flanker task across the morning following the low-GI breakfast is consistent with findings based on classroom observations in 6-7-year-olds who spent more time on task and demonstrated fewer lapses in attention following a low-GI breakfast compared with a high-GI breakfast ${ }^{(17)}$. Furthermore, in 9-16-year-olds completing an attentional task as part of the Cognitive Drug Research battery of cognitive tests, the accuracy of attention declined across the morning following a high-GI breakfast, but accuracy was better maintained following a low-GI breakfast ${ }^{(13)}$. These findings are in line with those of the present study, but the present study extends findings by using a more widely used test of attention (Flanker task) and focusing on adolescence, a time during which the frontal lobes thought to govern executive functions have been found to undergo a final bout of development ${ }^{(29)}$, whereas earlier studies focused on young people in general.

\section{Glycaemic and insulinaemic responses}

The glycaemic and insulinaemic responses to meals of differing GI have not been previously reported in adolescent populations. In the present study, adolescents exhibited a larger overall glycaemic response following a high-GI breakfast when compared with the low-GI breakfast (as determined by the IAUC). These findings are consistent with those in adult populations ${ }^{(30)}$. It has also been suggested that high-GI foods result in a higher insulinaemic response in adults ${ }^{(30)}$. However, in the adolescents tested in the present study, there was no difference in the overall insulinaemic response following the high-GI compared with the low-GI breakfast (as determined by the IAUC). A potential explanation for the similar insulinaemic response to the high- and low-GI trials is the matched milk content of the breakfasts, because of the well-documented insulinotropic effect of milk ${ }^{(31)}$. Therefore, the expected differences in insulinaemia (based on the findings in adults) could have been masked by the matched milk content of the meals.

\section{Summary and future research directions}

The main finding of the present study was that across a range of cognitive function tests, including the domains of working memory and attention, in adolescents, a low-GI breakfast enhanced both response times and accuracy later in the morning when compared with a high-GI breakfast and breakfast omission, particularly on the more cognitively demanding levels of the cognitive function tests. Furthermore, the lowGI breakfast produced a smaller overall glycaemic response when compared with the high-GI breakfast.

Overall, we conclude that a low-GI breakfast is more beneficial than both a high-GI breakfast and breakfast omission for cognitive function in adolescent school children across the school morning. However, further work is required to examine the optimal timing of breakfast and the effects of different macronutrients on cognitive function during the school morning. Furthermore, where possible, given the ethical constraints of working with young people, more detailed mechanistic work should be undertaken to suggest potential mechanisms for nutritional effects on cognitive function. 


\section{Acknowledgements}

S. B. C., S. B., M. L. N. and M. E. N. designed the research; S. B. C., M. L. N. and J. G. M. conducted the research; S. B. C. and S. B. analysed the data and S. B. C. and M. E. N. had primary responsibility for writing the manuscript. All authors read and approved the final manuscript. The authors would also like to thank Professor Clyde Williams and Professor Eef Hogervorst for their comments on the manuscript and Emma Batten for help with conducting the research. This study received no specific grant from any funding agency in the public, commercial or not-for-profit sectors. The authors have no conflicts of interest.

\section{References}

1. Smith AP, Kendrick A \& Maben A (1994) Effects of breakfast and caffeine on cognitive performance, mood and cardiovascular functioning. Appetite 22, 39-55.

2. Benton D, Slater O \& Donohoe RT (2001) The influence of breakfast and a snack on psychological functioning. Physiol Behav 74, 559-571.

3. Hoyland A, Dye L \& Lawton CL (2009) A systematic review of the effect of breakfast on the cognitive performance of children and adolescents. Nutr Res Rev 22, 220-243.

4. Widenhorn-Müller K, Hille K, Klenk J, et al. (2008) Influence of having breakfast on cognitive performance and mood in 13- to 20-year-old high school students: results of a crossover trial. Pediatrics 122, 279-284.

5. Cooper SB, Bandelow S \& Nevill ME (2011) Breakfast consumption and cognitive function in adolescent schoolchildren. Physiol Behav 103, 431-439.

6. Fischer K, Colombani PC, Langhans W, et al. (2001) Cognitive performance and its relationship with postprandial metabolic changes after ingestion of different macronutrients in the morning. BrJ Nutr 85, 393-405.

7. Gilsenan MB, de Bruin EA \& Dye L (2009) The influence of carbohydrate on cognitive performance: a critical evaluation from the perspective of glycaemic load. Br J Nutr $\mathbf{1 0 1}$ 941-949.

8. Benton D, Ruffin MP, Lassel T, et al. (2003) The delivery rate of dietary carbohydrates affects cognitive performance in both rats and humans. Psychopharmacology 166, 86-90.

9. Benton D \& Nabb S (2004) Breakfasts that release glucose at different speeds interact with previous alcohol intake to influence cognition and mood before and after lunch. Behav Neurosci 118, 936-943.

10. Nilsson A, Radeborg K \& Björck I (2009) Effects of differences in postprandial glycaemia on cognitive functions in healthy middle-aged subjects. Eur J Clin Nutr 63, 113-120.

11. Cromer BA, Tarnowski KT, Stein AM, et al. (1990) The school breakfast program and cognition in adolescents. J Dev Behav Pediatr 11, 295-300.

12. Kanarek R (1997) Psychological effects of snacks and altered meal frequency. BrJ Nutr 77, Suppl. 1, S105-S121.

13. Wesnes KA, Pincock C, Richardson D, et al. (2003) Breakfast reduces declines in attention and memory over the morning in schoolchildren. Appetite 41, 329-331.
14. Micha R, Rogers PJ \& Nelson M (2010) The glycaemic potency of breakfast and cognitive function in adolescent school children. Eur J Clin Nutr 64, 948-957.

15. Smith MA \& Foster JK (2008) The impact of a high versus a low glycaemic index breakfast cereal on verbal episodic memory in healthy adolescents. Nutr Neurosci 11, 219-227.

16. Brouns F, Bjorck I, Frayn KN, et al. (2005) Glycaemic index methodology. Nutr Res Rev 18, 145-171.

17. Benton D, Maconie A \& Williams C (2007) The influence of the glycaemic load of breakfast on the behaviour of children in school. Physiol Behav 92, 717-724.

18. Wolever TMS, Jenkins DJA, Jenkins AL, et al. (1991) The glycemic index: methodology and clinical implications. $\mathrm{Am}$ J Clin Nutr 54, 846-854.

19. Kuwa K, Nakayama T, Hoshino T, et al. (2001) Relationships of glucose concentrations in capillary whole blood, venous whole blood and venous plasma. Clin Chim Acta 307, $187-192$.

20. Wolever TMS (2003) Carbohydrate and the regulation of blood glucose and metabolism. Nutr Rev 61, S40-S48.

21. Wolever TMS \& Jenkins DJA (1986) The use of the glycaemic index in predicting blood glucose response to mixed meals. Am J Clin Nutr 43, 167-172.

22. Stroop JR (1935) The Stroop test. J Exp Psychol 18, 643-662.

23. Macleod CM (1991) Half a century of research on the Stroop effect: an integrative review. Psychol Bull 109, 163-203.

24. Sternberg S (1969) Memory-scanning: mental processes revealed by reaction-time experiments. Am Sci 57, 421-457.

25. Micha R, Rogers PJ \& Nelson M (2008) Glycaemic potency of breakfast predicts cognitive function and mood in adolescent schoolchildren. Proc Nutr Soc 67, E364.

26. Dye L, Lluch A \& Blundell JE (2000) Macronutrients and mental performance. Nutrition 16, 1021-1034.

27. Schulze MB, Liu S, Rimm ER, et al. (2004) Glycaemic index, glycaemic load, and dietary fibre intake and incidence of type 2 diabetes in younger and middle-aged women. $A m \mathrm{~J}$ Clin Nutr 80, 348-356.

28. Mahoney CR, Taylor HA, Kanarek RB, et al. (2005) Effect of breakfast composition on cognitive processes in elementary school children. Physiol Behav 85, 635-645.

29. Giedd JN, Blumenthal J, Jeffries NO, et al. (1999) Brain development during childhood and adolescence: a longitudinal MRI study. Nat Neurosci 2, 861-863.

30. Wolever TMS \& Bolognesi C (1996) Source and amount of carbohydrate affect postprandial glucose and insulin in normal subjects. J Nutr 126, 2798-2806.

31. Liljeberg HE \& Björk I (2001) Milk as a supplement to mixed meals may elevate postprandial insulinaemia. Eur J Clin Nutr 55, 994-999.

32. Cole TJ, Freeman JV \& Preece MA (1998) British 1990 growth reference centiles for weight, height, body mass index and head circumference fitted by maximum penalized likelihood. Stat Med 17, 407-429.

33. McCarthy HD, Jarrett KV \& Crawley HE (2001) Development of waist circumference percentiles in British children aged 5.0-16.9 y. Eur J Clin Nutr 55, 902-907.

34. Foster Powell K, Holt SHA \& Brand-Miller JC (2002) International table of glycaemic index and glycaemic load values: 2002. Am J Clin Nutr 76, 5-56. 